\title{
Retinal Redetachment After Pneumatic Retinopexy - The Importance of Case Selection
}

\author{
Muhammad Najmi K, Aida Zairani MZ, Mushawiahti M, Bastion MLC, Amin A ( $₫)$ \\ Department of Ophthalmology, Faculty of Medicine, Universiti Kebangsaan Malaysia Medical Centre, \\ Jalan Yaacob Latif, Bandar Tun Razak, 56000 Cheras, Kuala Lumpur, Malaysia.
}

\begin{abstract}
Pneumatic retinopexy is known as one of the treatment options for a specific type of retinal detachment. It is done in an office setting and may be the most cost-effective means of retinal reattachment surgery. Location and size of the retinal break remain as the major criteria for a successful outcome. We describe a case that fulfilled all except one major criteria for pneumatic retinopexy and underwent multiple procedures but failed. Fluctuation in the resolution of the retinal detachment such as in this particular case suggested possibility of early treatment failure.
\end{abstract}

Keywords: $\quad$ Floaters, pneumatic retinopexy, retinal detachment, scleral buckle, vitrectomy

\section{Correspondence:}

Amin bin Ahem. Department of Ophthalmology, Faculty of Medicine, Universiti Kebangsaan Malaysia Medical Centre, Jalan Yaacob Latif, Bandar Tun Razak, 56000 Cheras, Kuala Lumpur, Malaysia. Tel: +603-91455981 Fax: +603-91456673 E-mail: amin75sy@yahoo.com

\section{Introduction}

Pneumatic retinopexy is a procedure done to treat retinal detachment. It involves injecting expanding gas bubble into the vitreous cavity and securing retinal tear by using laser and/or cryopexy. The literature reports a variable success rate when compared to other conventional retinal reattachment surgeries. This procedure may be done in office setting thus remained as the most cost effective ways to treat retinal detachments.

\section{Case Report}

A 58-year-old Chinese gentleman with no known medical illness, presented with two weeks history of right eye superonasal visual field defect which progressed towards the centre, associated with flashes and floaters. There was no history of ocular trauma and patient was emmetropic. Examination revealed visual acuity of $6 / 60$ over the affected side with positive relative afferent pupillary defect. Slit-lamp examination of the right eye revealed normal anterior segment, presence of tobacco dusting with intraocular pressure of $7 \mathrm{mmHg}$. On fundal examination, there was a right eye rhegmatogeneous retinal detachment (Fig. 1) involving the macula with superior horse-shoe tear at 11 o'clock position (2 clock-hours size). The patient underwent right eye pneumatic retinopexy with an injection of $0.4 \mathrm{mls}$ of C3F8 gas (100\%) followed by indirect barricade laser around the tear two days later. He was compliant to the positioning instruction which was sit up with head tilt to the left. Fundal examination a week later showed satisfactory reduction in the area of detachment and early scarring of the laser marks. However, at 2 weeks posttreatment, residual retinal detachment was still present with small opening of the primary tear. In view of the positive response after the first procedure, he was subjected for a repeat pneumatic retinopexy with additional laser and injection of C3F8 $0.4 \mathrm{mls}(100 \%)$ to treat the small opening of the tear. Reattachment of the macula was achieved at 1-week post-procedure with residual inferior detachment. This was confirmed by the optical coherence tomography (OCT) (Fig. 2). Unfortunately, at 4 weeks post-pneumatic treatment, the macula redetached and the area of detachment remained fairly persistent with no signs of further 


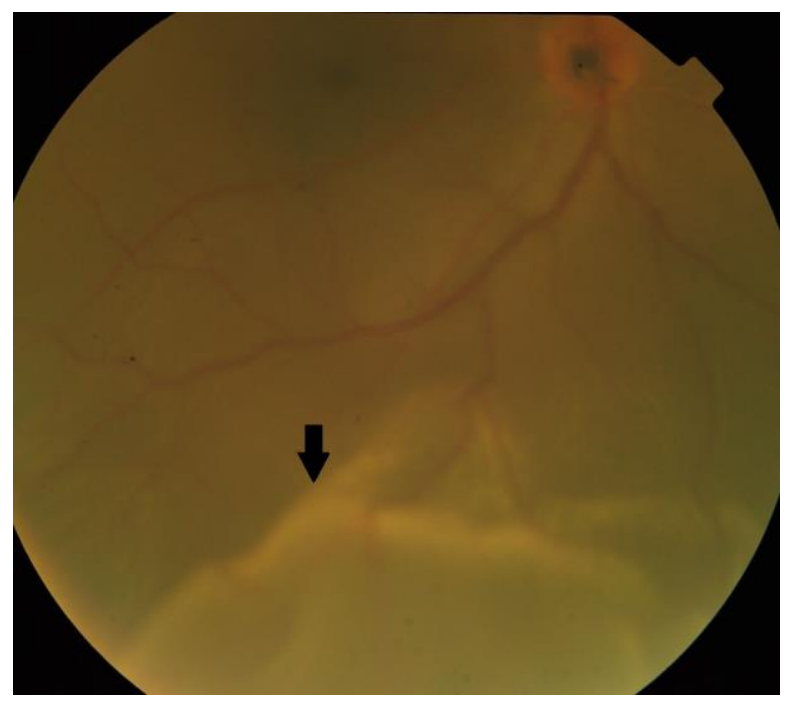

Figure 1: Fundus photograph of right eye showing retinal detachment with macula-off.

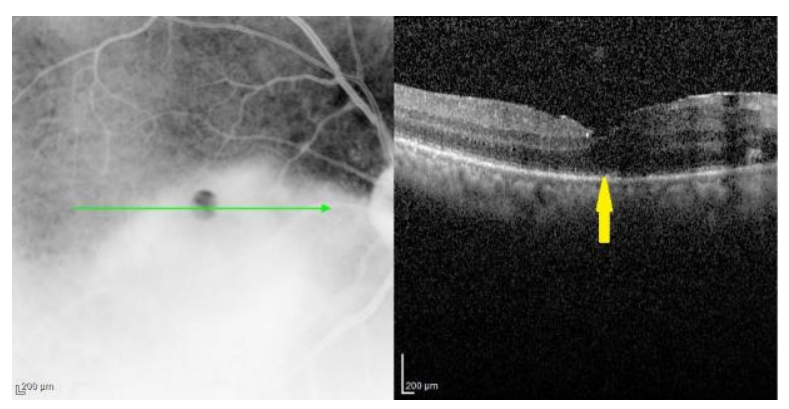

Figure 2: OCT showing macula reattachment after second pneumatic retinopexy treatment

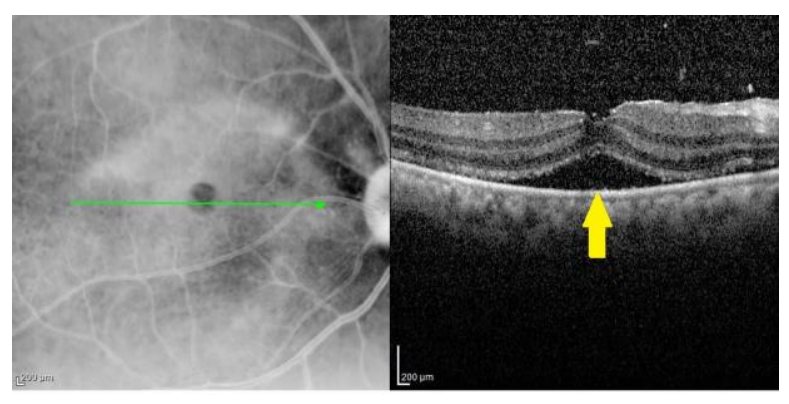

Figure 3: OCT showing macula re-detachment 4 weeks post-pneumatic treatment

resolution (Fig. 3). Eventually, 2 weeks after the redetachment, pars plana vitrectomy was performed with gas tamponade together with cataract extraction and intraocular lens implant. Intra-operatively, it was noted that the anterior lip of the retinal break did not close completely. There was no abnormal vitreoretinal adhesion or other retinal break noted. The retina remained flat at two months post-surgery with evidence of epiretinal membrane at the macula. His best corrected visual acuity was 6/36.

\section{Discussion}

The ultimate goals of primary rhegmatogenous retinal detachment repair are permanent anatomic success and restored vision. Pneumatic retinopexy is a minimally invasive and cost-effective means to achieve these goals. However, patient selection is a key factor to achieving a successful outcome with pneumatic retinopexy.

Among the main indications for pneumatic retinopexy are retinal break within the superior 8 clock hours (8 to 4 o'clock) with single or multiple breaks within 1 clock hour (1). The ocular media should be clear which provides good fundus view and the patient must be able to maintain positioning for one week after the procedure (2). Our patient fulfilled the criteria by having a superior retinal break with clear media and compliant to positioning. However, the retinal break is of 2 clock hours which is more than the indicated criteria.

A repeated pneumatic retinopexy following the first procedure is not an uncommon scenario. Half of pneumatic retinopexy cases needed reoperation within the first two weeks. This percentage increases to $70 \%$ within the first month and some reported reoperation rate as high as $87 \%$ at the end of three months (2). Despite that, repeated pneumatic retinopexy remained more cost-effective compared to scleral buckling (3). Another advantage of pneumatic retinopexy is that it does not adversely affect any subsequent surgery should this be required in the event of treatment failure.

Similarly, our patient was subjected to repeated pneumatic retinopexy in view of the positive response from the first procedure. This also suggests that eyes treated with pneumatic retinopexy should be followed very closely for the first few months after procedure. Serial funduscopic examinations during follow up with optical coherence tomography provides better analysis of the retinal detachment assessment and has to be taken into consideration during decision making. This would enable surgeon to evaluate signs of early treatment failure.

Pars plana vitrectomy with phacoemulsification was carried out to our patient as the right eye lens was noted to be cataractous with nuclear sclerosis. This might be secondary to multiple posterior segment pneumatic procedures carried out previously. Intraoperatively, it was noted that the anterior lip of the retinal tear was not properly closed despite two 
attempts of pneumatic retinopexy and additional laser supplement. This would be the main reason for recurrent redetachment for our patient. Failure of closure of the primary break despite two attempts of pneumatic retinopexy might be related to the larger size of the break in our patient.

There are a few identified prognostic factors which would influence the success of the surgery. In general, most surgeons hope to get a single-operation success. Pseudophakic retinal detachments are reported to have lower single-operation success rate compared to cases with phakic detachment (4). This is because pseudophakic detachments are usually associated with small peripheral breaks that are easily missed, especially in cases with hazy remaining peripheral lens capsule. More extensive detachments, larger break and more retinal breaks are associated with lower singleoperation success probably because they may suggest more vitreoretinal interface pathology (4-6).

At the same time, the surgical technique used during operation and post-procedure supplementary laser treatment does play a role on the final outcome. Compared with focal retinopexy, peripheral 3600 retinopexy improves single-operation success rate $20 \%$ more $(6,7)$.

The best corrected visual acuity obtained by our patient was $6 / 36$ at 2 months post-operation. However, visual recovery after retinal re-attachment surgery will continue up till 5 years post-operatively. Study by Kusaka et al. found that the best corrected visual acuities at 5 years were better by at least 2 lines than at 3 months in half of their study patients (8).

On the other hand, the relatively poor vision might be partially contributed by the presence of epiretinal membrane overlying the macula which was noted post-operatively. Another possible factor for the poor visual outcome would be the macula off retinal detachment itself. In experimental retinal detachments in owl monkeys, Machemer found that whenever the distance of the detachment between the pigment epithelial layer and the photoreceptors increases, there is increased photoreceptor cell degeneration (9). This will lead to irreversible nutritional damage to the macula and contribute to poor visual outcome despite anatomical reattachment post-operatively.

\section{References}

1. Hilton GF, Grizzard WS. Pneumatic retinopexy (a two-step outpatient operation without conjunctival incision). Ophthalmology 1986; 93(5): 626-41.

2. Tornambe PE, Hilton GF. Pneumatic retinopexy. A multicenter randomized controlled clinical trial comparing pneumatic retinopexy with scleral buckling. The Retinal Detachment Study Group. Ophthalmology 1989; 96(6): 772-83; discussion 784.

3. Han DP, Mohsin NC, Guse CE, Hartz A, Tarkanian CN. Comparison of pneumatic retinopexy and scleral buckling in the management of primary rhegmatogenous retinal detachment. Am J Ophthalmol 1998; 126(5): 658-68.

4. Grizzard WS, Hilton GF, Hammer ME, Taren D, Brinton DA. Pneumatic retinopexy failures. Cause, prevention, timing, and management. Ophthalmology 1995; 102(6): 929-36.

5. Kulkarni KM, Roth DB, Prenner JL. Current visual and anatomic outcomes of pneumatic retinopexy. Retina 2007; 27(8): 1065-70.

6. Zaidi AA, Alvarado R, Irvine A. Pneumatic retinopexy: success rate and complications. $\mathrm{Br} \mathbf{J}$ Ophthalmol 2006; 90(4): 427-28.

7. Mudvari SS, Ravage ZB, Rezaei KA. Retinal detachment after primary pneumatic retinopexy. Retina 2009; 29(10): 1474-78.

8. Kusaka S, Toshino A, Ohashi Y, Sakaue E. Long-term visual recovery after scleral buckling for macular-off retinal detachments. Jpn J Ophthalmol 1998; 42(3): 218-22.

9. Machemer R. Experimental retinal detachment in the owl monkey. II. Histology of retina and pigment epithelium. Am J Ophthalmol 1968; 66(3): 396-410. 